\title{
Lithium-ion Battery Electro-thermal Model and Its Application in the Numerical Simulation of Short Circuit Experiment
}

\author{
Chengtao Lin ${ }^{1}$, Can $\mathrm{Cui}^{2}$, Xiaotian $\mathrm{Xu}^{3}$ \\ ${ }^{I}$ The State Key Laboratory of Automotive Safety and Energy, Tsinghua University, Beijing, China, \\ 100084,lct@tsinghua.edu.cn
}

\begin{abstract}
As a key issue in EVs (Electric Vehicles) development, Li-ion battery's thermal safety is focused on in which the thermal abuse model is an important tool. This paper has established a 3-D electro-thermal model taking side reaction into account, in order to study on MGL100Ah lithium manganite battery's safety issue. This paper firstly states the mechanism of heat generation, types of thermal model, and the mesh model, then analyzes the simulation on the model under the short circuit condition, one of thermal abuse condition. On one hand, the paper verifies the liability of the model, so as to apply the model on other thermal abuse condition in the future; on the other hand, the paper discusses in detail the internal temperature distribution, voltage and current changes under the short circuit condition. Analyzing this typical safety issue in details, this paper can help reduce cost, raise design efficiency and avoid thermal abuse in future development of battery.
\end{abstract}

Keywords: Lithium battery, Thermal Management, Short Circuit, Modeling

\section{Introduction}

The vehicle industry has developed rapidly and become biggest industry in many large industrial countries since the 21 st century. In 2013, many Chinese cities were threatened by fog and haze, which can raise probability of lung cancer and harm people's health. Traditional fuel vehicle emission is considered to be the culprit of that poor weather. Nowadays non-renewable energy like petroleum is going to the end day by day. Due to the world's sustainable strategy, as a result of solving both energy and pollution problems, EV (Electric Vehicle) is under lots of studies in many countries. Storage battery has won high attention as is the "heart" of EV. Differing from ordinary cell phone or laptop battery, the large-scale battery in EV must possess 3 main performances: 1) Good safety; 2)
High specific energy and specific power; 3) Low cost. These are the basic elements for application and promotion of future EV. Li-ion battery is one of the most high-potential energy sources in the future, which is the high-quality battery satisfying any working condition of EVs.

Li-ion batteries have high specific energy and specific power, but they are doubted in safety for many times. In 1995 and 1997, there were two li-ion battery explosion accidents in Japan which caused large loss of property and personal injuries. Every accident revealed problems that need to be solved related to li-ion safety.

Many experiments and analyses tell that under short circuit, large current over-charge and high temperature etc. conditions the li-ion battery was in unpredictable danger. In order to discovery these thermal abuse condition, nowadays, each country has 
built unified battery safety standard, for instant, IEC, JIS, GB, EN, etc. In these standards, thermal abuse behaviors are stated and standardized generally, and they are used to test li-ion batteries safety.

Many researchers from different institutes has studied much on Li-ion battery's abuse: Hatchard et al mainly focused on small-scale Li-ion batteries (e.g. 18650 cylinder battery) and their abuse simulation; Kim et al from National renewable energy laboratory extended that into $3 \mathrm{D}$ abuse model and carried out simulation in depth on oven test. However, in this field the study on large-scale battery has not been carried out yet, especially under external short circuit condition.

This paper will build a 3D electro-thermal model which can simulate thermal abuse performance of 100Ah lithium manganate battery well. Combining experiments with simulations, this paper mainly studies on the safety issue under short circuit condition, and predicting the thermal behavior under thus condition. Using this model we expect that the cost in battery development will be decreased and the design efficiency will be raised, what's more, achieve the goal of reducing thermal abuse by controlling temperature.

\section{Battery Introduction}

\subsection{Battery structure and size}

The object of the study is MGL100Ah lithium manganite battery produced by Citic Guoan MGL Power Sources Co, Ltd. It's a large-scale storage battery fitting Evs. In micro view, the battery is arranged in stacks. One single stack unit contains 5 layers: current collector on positive electrode, positive electrode, separator, negative electrode and current collector on negative electrode, as is shown in Fig.2. The whole battery is made up of 88 fairly same stacks.

In macro view, MGL100Ah battery is $295 \mathrm{~mm}(\mathrm{X})$ x $320 \mathrm{~mm}(\mathrm{Y})$ x $22 \mathrm{~mm}(\mathrm{Z})$. The sizes of every detail are shown in Fig.3 and Fig.4.

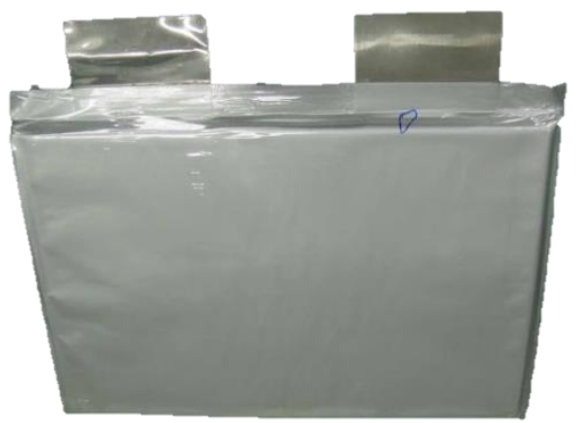

Fig.1 MGL100Ah Li-on battery

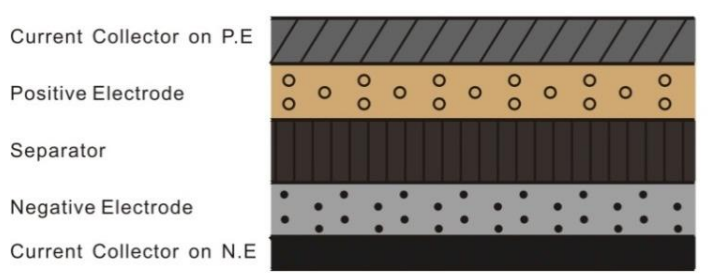

Fig.2 Battery unit's layer model

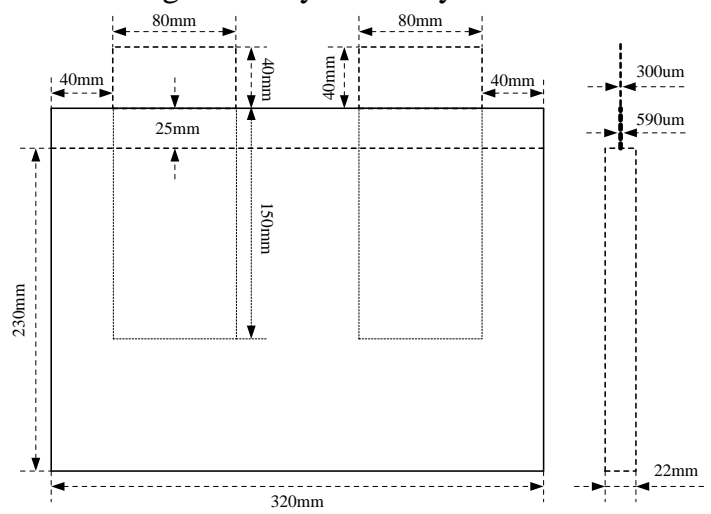

Fig.3 MGL100Ah battery's spatial size

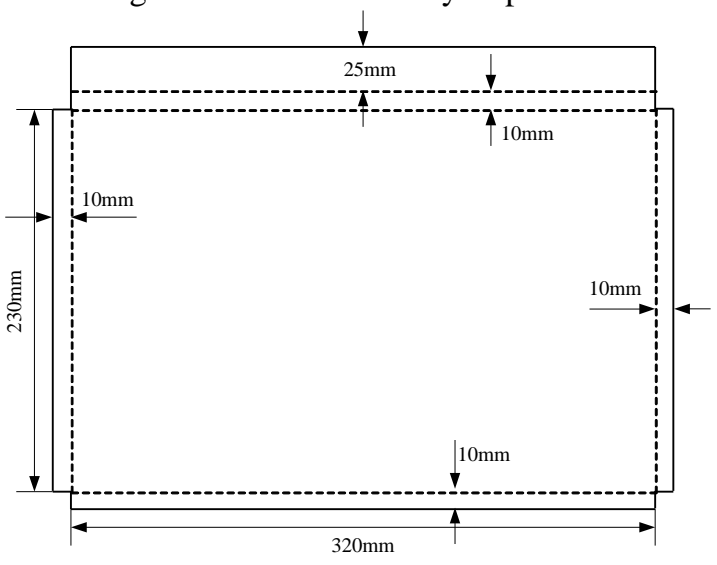

Fig.4 MGL100Ah battery shell's spatial size

\subsection{Battery materials}

Current collector is made with homogeneous and isotropous metal, aluminum by the positive side and copper by the negative side. Positive electrode material is $\mathrm{LiMnO} 3$ while negative one is graphite. According to the pre-study, the separators are filled with electrolyte, where separator material is $\mathrm{PE} / \mathrm{PP} / \mathrm{PE}$, electrolyte is LiPF6, organic solvent is $\mathrm{DEC}+\mathrm{DMC}+\mathrm{EC}$; positive electrode mainly contains positive active material, binder and electrolyte while negative electrode contains negative active material, binder and electrolyte. According to integrative compute and experiments, this paper obtained the thermal physical parameter of the three parts mentioned above. Next, all these part together will be called one unit. Besides, one positive and one negative lug are extended out from the two kinds of current collectors, and these two lugs are connected with external circuit. 


\subsection{Battery electric performance}

After HPPC (Hybrid Pulse Power Characteristic) experiment, we collected the battery's OCV (Open Circuit Voltage) and equivalent internal resistance changes with SOC (State of Charge), which are shown in Fig.5 and Fig.6.

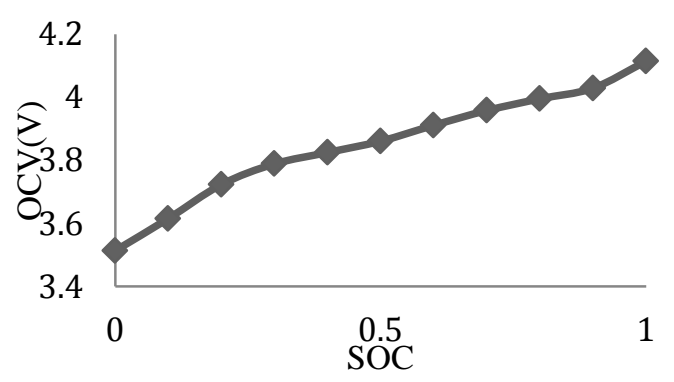

Fig.5 OCV changes with SOC

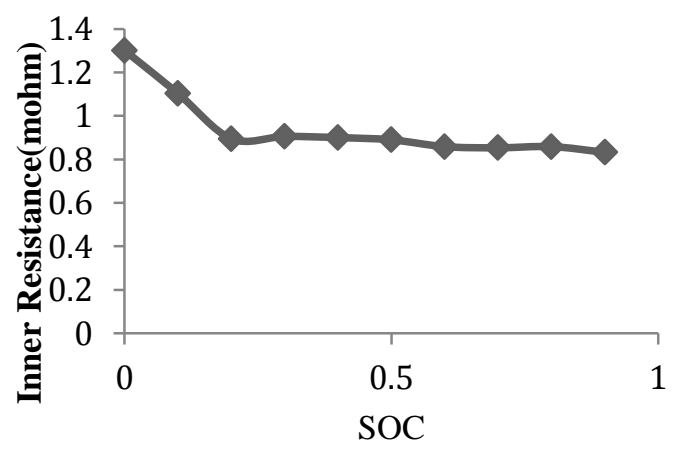

Fig.6 battery's internal resistance changes with SOC

The OCV will grow when SOC grows, while the equivalent internal resistance will descend. Through fitting calculation, the paper obtained 2 fitted curves, which are shown in Eq.1 and Eq.2. $\mathrm{OCV}=0.8649 * \mathrm{SOC}^{3}-1.5478 * \mathrm{SOC}^{2}+$ $1.2815 * \mathrm{SOC}+3.5117$ $R_{i}=-2.6 * S O C^{3}+4.4 * S O C^{2}-2.4 * S O C+$ Eq. 1 1.3

Eq. 2

\section{Establishment of 3D electro- thermal model}

\subsection{Thermal physical parameters}

Through experiments and research, this paper obtained the thermal physical parameters, they are shown in Table.1.

\subsection{Mesh model}

The mesh model for follow-up numerical simulation is shown in Fig.7, which is made up of triangle meshes. The very thin parts are meshed with adjusted laxity degree and the whole model consists of 67836 grids.

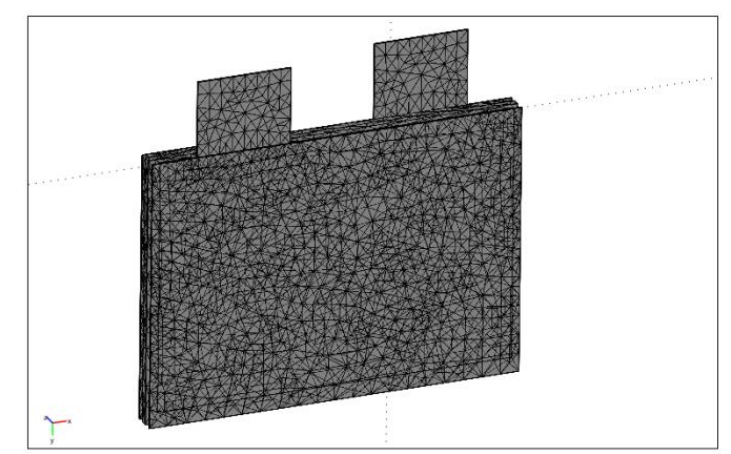

Fig.7 Battery’s mesh model

\subsection{Thermal model}

\subsubsection{Heat equilibrium equation}

The transient 3D energy transfer formula in Li-ion battery is shown in Eq.3, and this formula can fit any tiny unit element in the battery, where $\rho$ is the density of the unit, $C$ is equivalent specific heat capacity, $T$ is temperature, $k$ is equivalent heat conductivity coefficient (which is a matrix in 3D model as shown in Eq.4), $Q$ is heat generation rate, and $q_{\text {dis }}$ is surface's heat diffusion rate.

$$
\begin{gathered}
\rho C \frac{\partial T}{\partial t}=\nabla g(k \nabla T)+Q-q_{d i s} \\
k=\left[\begin{array}{ccc}
k_{x x} & 0 & 0 \\
0 & k_{y y} & 0 \\
0 & 0 & k_{z z}
\end{array}\right]
\end{gathered}
$$

One popular heat generation model is raised by $\mathrm{D}$. Bernardi in $1985^{[i]}$ as shown in Eq.5, which can explain the whole battery's heat generation condition, where $Q$ is the battery's heat generation rate, $Q_{I}$ is irreversible heat generation rate, $\mathrm{Q}_{\mathrm{r}}$ is reversible heat generation rate, $I$ is the current through the battery, $E$ is the equilibrium voltage, $\mathrm{U}$ is the working voltage, $\mathrm{T}$ is its absolute temperature.

$$
\mathrm{Q}=\mathrm{Q}_{\mathrm{i}}+\mathrm{Q}_{\mathrm{r}}=\mathrm{I}(\mathrm{E}-\mathrm{U})-\mathrm{IT} \frac{\mathrm{dE}}{\mathrm{dT}} \quad \mathrm{Eq} .5
$$

In order to get a more accurate model, we take side reaction heat generation into consider, it becomes Eq.6 which is similar to Sato heat generation rate model[1]:

$$
\begin{aligned}
\mathrm{Q} & =\mathrm{Q}_{\mathrm{r}}+\mathrm{Q}_{\mathrm{p}}+\mathrm{Q}_{\mathrm{s}}+\mathrm{Q}_{\mathrm{j}} \\
& =\mathrm{nFT}\left(\frac{\partial \mathrm{E}_{0}}{\partial \mathrm{T}}\right)-\mathrm{nF}\left(\mathrm{E}_{0}-\mathrm{U}_{\mathrm{L}}\right)+\mathrm{Q}_{\mathrm{s}}+\mathrm{Q}_{\mathrm{j}} \quad \text { Eq.6 }
\end{aligned}
$$

Where $Q_{r}$ is the heat from battery reaction, $Q_{j}$ is the Joule heat, $Q_{p}$ is the polarization heat and $Q_{s}$ is the side reaction heat.

Unit's heat diffusion rate $\mathrm{q}_{\text {dis }}$ can be divided into two parts: the convection heat transfer rate $\mathrm{q}_{\text {conv }}$ and the radiative heat transfer rateq ${ }_{\text {rad }}$, as shown in Eq.7.

$$
q_{\text {dis }}=q_{\text {conv }}+q_{\text {rad }} \quad \text { Eq. } 7
$$

Equation of the convection heat transfer is shown in Eq.8, where $\mathrm{h}$ is the surface's heat transfer 
Table.1 Temophysical parameters in every layer material

\begin{tabular}{|c|c|c|c|c|c|}
\hline & $\begin{array}{c}\text { Current } \\
\text { collector of P.E }\end{array}$ & Positive Electrode & Separator & $\begin{array}{l}\text { Negative } \\
\text { electrode }\end{array}$ & $\begin{array}{c}\text { Current collector of } \\
\text { N.E }\end{array}$ \\
\hline Material & aluminum & lithium manganate & $\mathrm{PP}|\mathrm{PE}| \mathrm{PP}$ & graphite & copper \\
\hline Density (kg.m-3) & $2.70 \times 10^{3}$ & $2.80 \times 10^{3}$ & $1.30 \times 10^{3}$ & $1.20 \times 10^{3}$ & $8.92 \times 10^{3}$ \\
\hline $\begin{array}{c}\text { Specific heat capacity } \\
(\text { J.kg-1.K-1) }\end{array}$ & 880 & 839 & 1980 & 1064 & 390 \\
\hline $\begin{array}{c}\text { Thermal conductivity } \\
\text { (W.m-1.K-1) }\end{array}$ & 237 & 3.9 & 0.6 & 3.3 & 397 \\
\hline Resistivity in $\mathrm{X}(\Omega . \mathrm{m})$ & $2.83 \times 10^{-8}$ & 33.2 & $\infty$ & 1.26 & $1.75 \times 10^{-8}$ \\
\hline Resistivity in $\mathrm{Y}(\Omega . \mathrm{m})$ & $2.83 \times 10^{-8}$ & 33.2 & $\infty$ & 1.26 & $1.75 \times 10^{-8}$ \\
\hline Resistivity in $\mathrm{Z}(\Omega . \mathrm{m})$ & $2.83 \times 10^{-8}$ & 33.2 & 0.25 & 1.26 & $1.75 \times 10^{-8}$ \\
\hline
\end{tabular}

coefficient, $\mathrm{A}$ is the surface area, $\mathrm{T}$ is the surface's temperature, $\mathrm{T}_{\text {air }}$ is the atmosphere temperature. Equation of the radiative heat transfer is shown in Eq.9, where $\sigma$ is Stefan-Boltzmann constant, $\varepsilon$ is emissivity, $\mathrm{T}_{\infty}$ is the ambient temperature. The $\mathrm{q}_{\text {rad }}$ is always much less than $\mathrm{q}_{\text {conv }}$, so the term $\mathrm{q}_{\mathrm{rad}}$ can be ignored except when it's in an oven test.

$$
\begin{array}{lc}
\mathrm{Q}_{\text {conv }}=\mathrm{hA}\left(\mathrm{T}-\mathrm{T}_{\mathrm{air}}\right) & \text { Eq. } 8 \\
q_{\text {rad }}=\sigma \varepsilon A\left(T^{4}-T_{\infty}^{4}\right) & \text { Eq. } 9
\end{array}
$$

\subsubsection{Side reaction heat generation model}

Side reactions mainly consist of SEI (Solid Electrolyte Interface) decomposition, Negativesolvent reaction, positive-solvent reaction and electrolyte decomposition. Batteries under special condition may cause different degrees of side reactions. So the heat generation rate from side reactions need to be taken into account in special working conditions. Based on the studies from National Renewable Energy Laboratory's researcher Gi-Heon Kim et al ${ }^{[i i]}$, we hereby expand the equations into our Li-ion batteries. Following the formulation of Hatchard et $\mathrm{al}^{[\mathrm{iii}]}$, the 4 side reactions can be expressed in the following equations:

(4) SEI (Solid electrolyte interface) decomposition reaction:

$$
\begin{array}{ll}
R_{\text {sei }}\left(T, c_{s e i}\right)=A_{\text {sei }} \exp \left[-\frac{E_{a, s e i}}{R T}\right] c_{\text {sei }}^{m_{\text {sei }}} & \text { Eq. } 10 \\
S_{s e i}=H_{\text {sei }} W_{c} R_{\text {sei }} & \text { Eq. } 11 \\
\frac{d c_{s e i}}{d t}=-R_{\text {sei }} & \text { Eq. } 12
\end{array}
$$

where $\mathrm{c}_{\text {sei }}$ is the dimensionless amount of metastable SEI, $\mathrm{R}\left(\mathrm{s}^{-1}\right)$ and $\mathrm{A}\left(\mathrm{s}^{-1}\right)$ are frequency parameters, $\mathrm{E}_{\mathrm{a}}\left(\mathrm{J} \cdot \mathrm{mol}^{-1}\right)$ is the activation energy of the reaction, $\mathrm{H}_{\text {sei }}\left(\mathrm{J} \cdot \mathrm{g}^{-1}\right)$ is the SEI-decomposition heat release, $\mathrm{W}_{\mathrm{c}}\left(\mathrm{g} \cdot \mathrm{m}^{-3}\right)$ is the volume-specific carbon content.

(2) Negative-solvent reaction:

$$
\begin{aligned}
& \mathrm{R}_{\text {ne }}\left(\mathrm{T}, \mathrm{c}_{\text {neg }}, \mathrm{t}_{\text {sei }}\right)= \\
& \mathrm{A}_{\text {ne }}\left(\frac{\mathrm{t}_{\text {sei,ref }}}{\mathrm{t}_{\text {sei }}}\right) \mathrm{c}_{\text {neg, }} \exp \left[-\frac{E_{\mathrm{a}, \text { ne }}}{\mathrm{RT}}\right] \\
& \mathrm{S}_{\text {ne }} \stackrel{=}{=} \mathrm{H}_{\text {ne }} \mathrm{W}_{\mathrm{c}} \mathrm{R}_{\text {ne }}
\end{aligned}
$$

$\frac{\mathrm{dc} \text { neg }}{\mathrm{dt}}=-\mathrm{R}_{\text {ne }}$

Eq. 15

where $c_{\text {neg }}$ and $t_{\text {sei }}$ is the dimensionless amount of lithium in the carbon/SEI, $\mathrm{R}\left(\mathrm{s}^{-1}\right)$ and $\mathrm{A}\left(\mathrm{s}^{-1}\right)$ are frequency parameters, $\mathrm{E}_{\mathrm{a}}\left(\mathrm{J} \cdot \mathrm{mol}^{-1}\right)$ is the activation energy of the reaction, $\mathrm{H}_{\mathrm{ne}}\left(\mathrm{J} \cdot \mathrm{g}^{-1}\right)$ is the heat release, $\mathrm{W}_{\mathrm{c}}\left(\mathrm{g} \cdot \mathrm{m}^{-3}\right)$ is the specific carbon content.

(3)Positive-solvent reaction:

$$
\mathrm{R}_{\mathrm{pe}}(\mathrm{T}, \alpha)=\mathrm{A}_{\mathrm{pe}} \alpha^{\mathrm{m}_{\mathrm{pe}, \mathrm{p} 1}}(1-\alpha)^{\mathrm{m}_{\mathrm{pe}, \mathrm{p} 2} \exp \left[-\frac{\mathrm{E}_{\mathrm{a}, \mathrm{pe}}}{\mathrm{RT}}\right]}
$$

Eq.16

$$
\begin{array}{ll}
\mathrm{S}_{\mathrm{pe}}=\mathrm{H}_{\mathrm{pe}} \mathrm{W}_{\mathrm{p}} \mathrm{R}_{\mathrm{pe}} & \text { Eq. } 17 \\
\frac{\mathrm{d} \alpha}{\mathrm{dt}}=\mathrm{R}_{\mathrm{pe}} & \text { Eq. } 18
\end{array}
$$

where $\alpha$ is the conversion degree, $\mathrm{R}\left(\mathrm{s}^{-1}\right)$ and $\mathrm{A}\left(\mathrm{s}^{-1}\right)$ are frequency parameters, $\mathrm{E}_{\mathrm{a}}\left(\mathrm{J} \cdot \mathrm{mol}^{-1}\right)$ is the activation energy of the reaction, $\mathrm{H}_{\mathrm{pe}}\left(\mathrm{J} \cdot \mathrm{g}^{-1}\right)$ is the heat release, $W_{p}\left(g \cdot \mathrm{m}^{-3}\right)$ is the specific positive-active content.

(4)Electrolyte decomposition:

$$
\begin{array}{lc}
R_{e}\left(T, c_{e}\right)=A_{c} \exp \left[-\frac{E_{a, e}}{R T}\right] c_{e}^{m_{e}} & \text { Eq. } 19 \\
S_{e l e}=H_{e} W_{e} R_{e} & \text { Eq. } 20 \\
\frac{\mathrm{dc}_{\mathrm{e}}}{\mathrm{dt}}=-\mathrm{R}_{\mathrm{e}} & \text { Eq. } 21
\end{array}
$$

where $c_{e}$ is the dimensionless concentration, $R\left(s^{-1}\right)$ and $\mathrm{A}\left(\mathrm{s}^{-1}\right)$ are frequency parameters, $\mathrm{E}_{\mathrm{a}}\left(\mathrm{J} \cdot \mathrm{mol}^{-1}\right)$ is the activation energy of the reaction, $\mathrm{H}_{\mathrm{e}}\left(\mathrm{J} \cdot \mathrm{g}^{-1}\right)$ is the heat release, $\mathrm{W}_{\mathrm{e}}\left(\mathrm{g} \cdot \mathrm{m}^{-3}\right)$ is the specific electrolyte content.

\section{Experiment}

This paper mainly discusses about external short circuit numerical simulation which hasn't been studied much on. For follow-up different conditions simulation, the experiment is designed for knowing about the battery's thermal behavior as well as comparing with following simulation results. First, we need to obtain the real condition results under external short circuit. Following is the process of the experiment.

\subsection{Experiment process}

(1)Device ready: Test devices, ensure reliability and safety; 
(2)Design for battery data collector: As is shown in Fig.8, set 6 data collectors and connect the device with the battery;

(3)Start: Start collecting data, close the circuit to short-circuit the battery, keep on 10min after the voltage stop dropping;

(4)Finish: Power off devices, stop collecting.

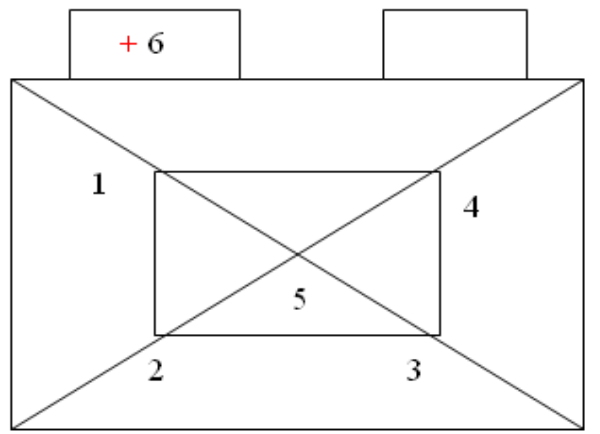

Fig.8 Short circuit Experiment's data collectors

First of all, there is no significant physical change observed from outside look, no plumping or exploding, as is shown in Fig.9.

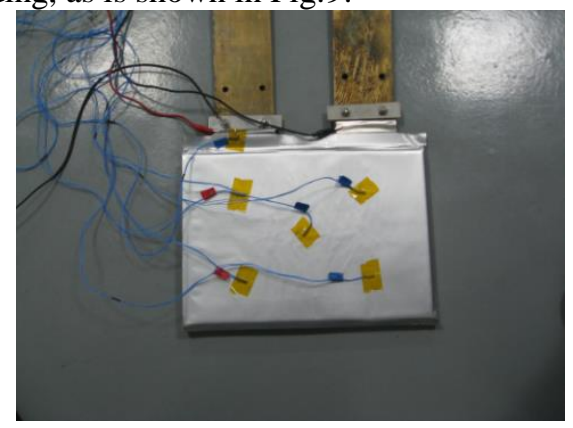

Fig.9 (a) Before short circuit

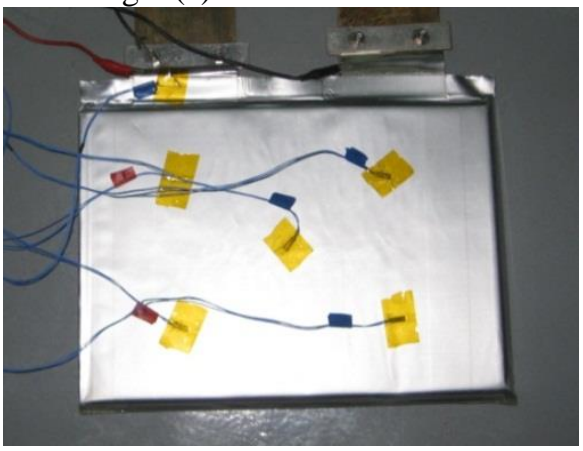

Fig.9 (b) After short circuit

\subsection{Experiment results and analysis}

Through processing the collected data, the current and voltage changes with time are shown in Fig.10. The whole short circuit period lasted until about 7 second when the voltage drop to $0 \mathrm{~V}$. The maximum current is nearly $1800 \mathrm{~A}$.

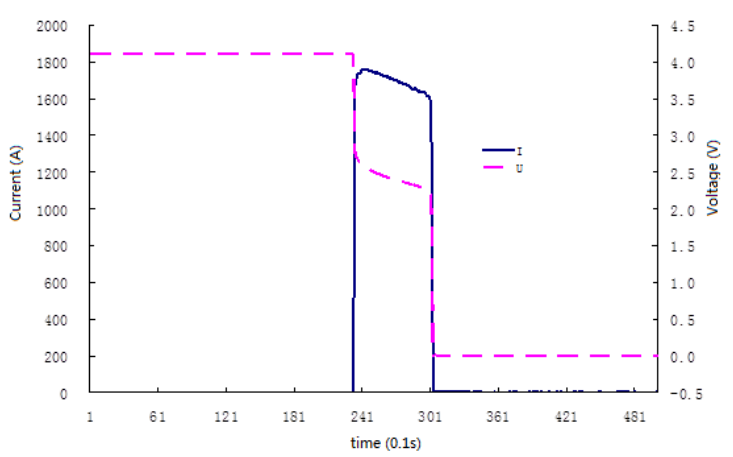

Fig.10 Voltage and current changes during short circuit

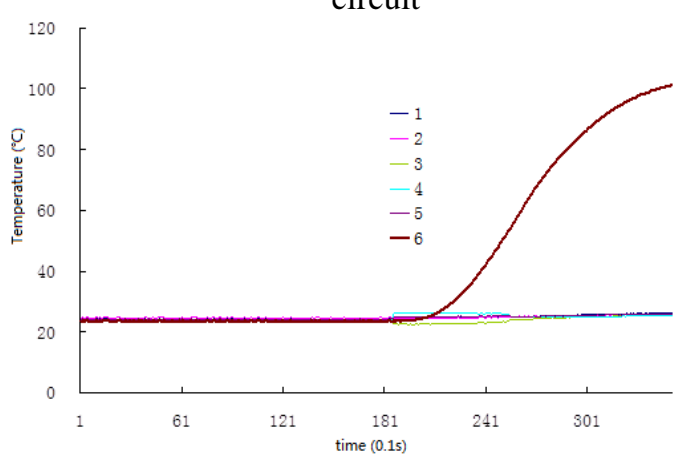

Fig.11 Temperature changes during short circuit

The temperature changes are shown in Fig.11. Since the short circuit period was short, the battery body temperature rise was small, no significant physical changes occurred on the body. But the lug temperature rise was relatively huge, even after short circuit stopped; the lug's temperature still rose fast for a while. It was because the temperature of the furthest point of the lug and of external circuit was far higher than point 6 , so that they could convey heat to the lug even if the short circuit stopped. Thus, the lug's highest local temperature was higher than that in the data, and it occurred on the edge of the lug where connected with external circuit. The local high temperature caused metal fusing which stopped the short circuit period. It is obvious that some safety problems may lie in this high local temperature status.

In general, the short circuit period, temperature changes, current and voltage changes were characteristics stating battery external short circuit, and they provided the comparison source.

\section{Simulation results and analysis}

\subsection{Simulation results}

Through building 3D electro-thermal model, this paper carried out the numerical simulation on battery external short circuit. During simulation, this period was $7.2 \mathrm{~s}$, and then the voltage suddenly dropped to $0 \mathrm{~V}$.

We obtained the current change during the simulation which is shown in Fig.12. The maximum 
of current was nearly $1800 \mathrm{~A}$ at the beginning and then slowly went down, that fitted the experiment data well.

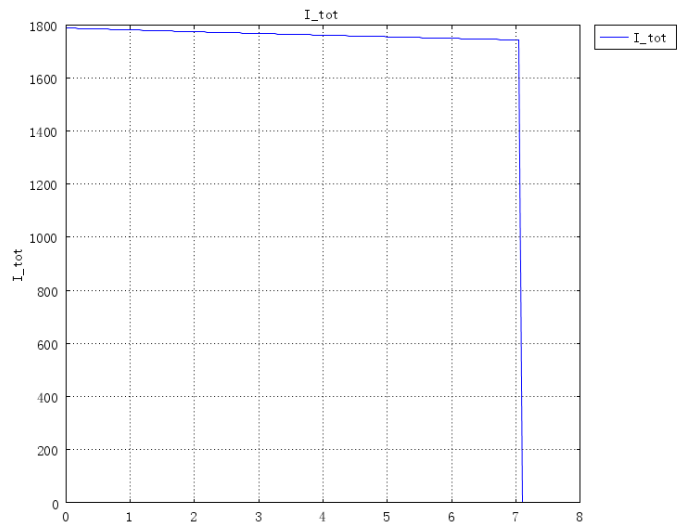

Fig.12 Current during short circuit



Fig.13 Temperature distribution after short circuit

Using postprocess program, we obtained the temperature distribution of the battery. Fig.13 shows the temperature distribution at the time of $7.2 \mathrm{~s}$. It's easy to identify that 2 lugs have biggest temperature rises while the body has no significant change in temperature. The highest temperature point is at the end of the positive lug (Aluminum) where it fused. The process resembles the experiment very much.

\subsection{Simulation results analysis}

Firstly, the temperature comparison between data collected and simulation data at the same point, as is shown in Fig.14. Center_Lab and center_Sim show temperature curves at point 5 from experiment and simulation respectively, meanwhile, lug_Lab and lug_Sim show temperature curves at point 6 from experiment and simulation respectively. Both simulation curves' trends are very similar to that in experiment, and also the lugs temperature rise is nearly the same, far more than the temperature rise in the battery body. The only difference is the temperature rise rate is not same on the lugs. The error comes from external circuit device's heat transfer, for there is only the battery built in the simulation without external circuit devices. Under this condition, the external circuit's heat transfer can hardly influence the temperature of the battery body, but can only influence the edge of the lugs. Due to the different heat generation rate between lugs and external circuit devices, the gradients shown in Fig.14 of 2 lug curves are not exactly the same. This error is in reasonable range.

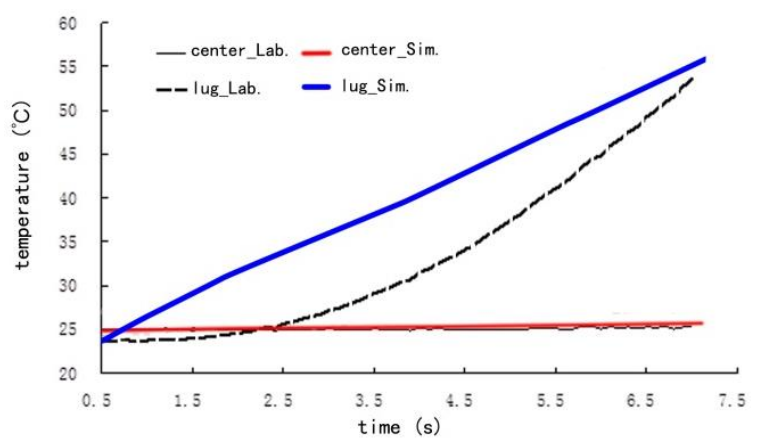

Fig.14 Surface temperature changes during short circuit

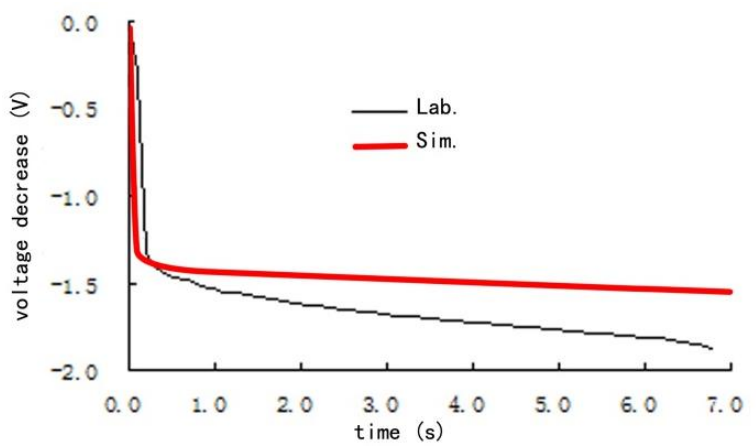

Fig.15 Voltage drop during short circuit

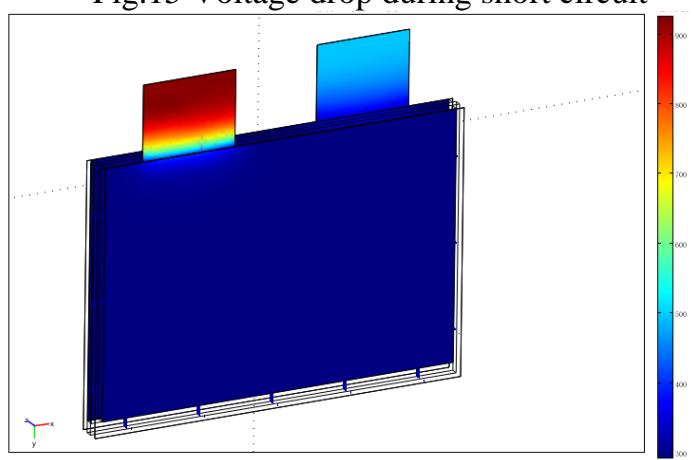

Fig.16 Inner temperature distribution at $7.2 \mathrm{~s}$

The voltage change is shown in Fig.15. The trends are basically same but the experiment value is a little smaller. To analyze this little error, we start from the metal's resistance increasing with the temperature rising. So every point in lugs has different increase in resistance, the further from the battery body the greater change it has, namely, the internal resistance of the whole battery is getting bigger. Thus the external circuit's voltage is getting smaller. However, the internal resistance we used in simulation includes 
the lugs' resistance ignoring the little change in lugs resistance which finally caused the little difference.

The side reactions are not significant, as is shown in Fig.16, but they have indeed occurred a little, as is shown in Fig.17, where hr_sei expresses the heat generation of SEI decomposition, hr_ne expresses the heat generation of Negative-solvent reaction, hr_pe expresses the heat generation of Positivesolvent reaction, hr_ele expresses the heat generation of Electrolyte decomposition, and all the units are translated into temperature rise of the whole battery $(\mathrm{K} / \mathrm{min})$. It is shown that these 4 side reactions can be described well by this model, however, the level of reactions are very low due to the very small temperature rise in the battery body. Only SEI decomposition and Negative-solvent reaction started a little. Compared with that in oven test simulation, these can be ignored.

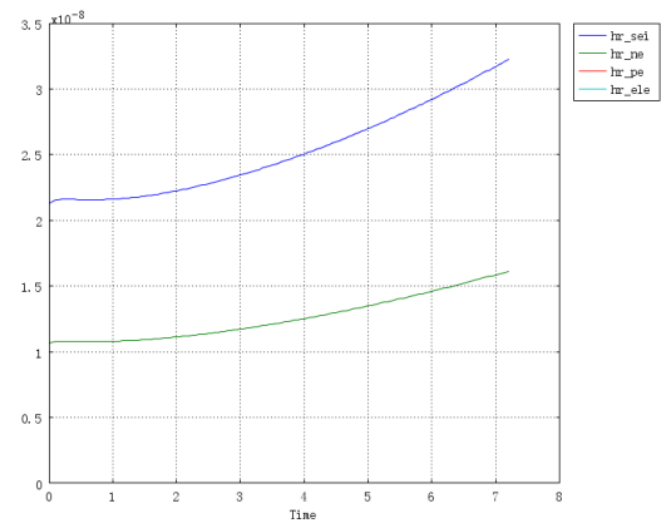

Fig. 17 Heat generation rate of side reactions

Thus, the main heat source is Joule heat from circuit under short circuit condition. The inner temperature rise is little so that side reactions' activities are rather low. No side reaction occur, it is good for battery safety.

In general, the 3D electro-thermal model established here can simulate the physical field's changes well, and the errors are in reasonable range without any problems in simulation. Results from both experiment and simulation show that the lugs are playing important roles under external short circuit condition. The local high temperature may bring danger. To avoid the unnecessary loss of property, suggestions are made: 1 . Set the lugs as metal with low melting point which can play as a fuse; 2 . Set additional short circuit protection circuit.

\section{Conclusion}

This paper built a 3D electro-thermal model according to MGL100Ah lithium manganite battery which is commonly used. And the paper carried out the experiment and simulation under external short circuit one of the typical thermal abuse conditions. The results show the study method is feasible and tells:
1) 3D model can help know deeply and directly about the temperature distribution in the battery under any working condition, this is an effective way to evaluate battery's safety.

2) The simulation results in this paper basically fit the real experiment's data, including potential dangers. The model has great application value in guiding the battery designs.

3) In the very short time period of external short circuit, the current is huge, thus the heat impulse is huge on lugs which are potential safety hazard points, while the internal thermal safety mainly lies in battery materials and structure.

4) The model studied in this paper can be applied in other thermal abuse conditions (e.g. over charge, oven test, etc.). This model will make great contributions in methods to the future Li-ion battery thermal management technology in depth.

\section{Acknowledgements}

Gratitude must be extended to those who had ever helped in the study of this paper: NSFC project (51006056), Independent funded project of Tsinghua University (2011THZ01004), Professor Guangyu Tian and Jiangbo Zhang from Tsinghua University, staffs of Citic Guoan MGL Power Sources Co, Ltd.

\section{References}

[1] D. Bernardi, E. Pawlikowski, J. Newman. A General Energy Balance for Battery Systems. J. Electrochem. Soc., 1985, 132(1): 5-12.

[2] Sato, N., Thermal behavior analysis of lithiumion batteries for electric and hybrid vehicles. power sources, 2001(77): p. 71

[3] Gi-Heon Kim, Ahmad Pesaran, Robert Spotnitz. A three-dimensional thermal abuse model for lithium-ion cells. Journal of Power Sources 170 (2007) 476-489

[4] T.D. Hatchard, D.D. MacNeil, A. Basu, J.R. Dahn, J. Electrochem. Soc., 148 (7) (2001) A755A761.

\section{Authors}

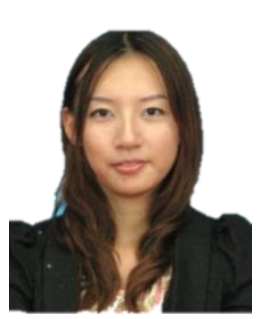

Can Cui, Postgraduate

The State Key Laboratory of Automotive Safety and Energy

Department of Automotive Engineering, Tsinghua University Beijing 100084, P.R.China Tel: +86 1381-159-1351, E-mail: cuican0118@139.com

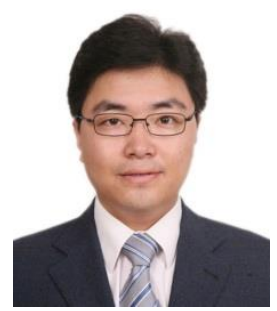

Chengtao Lin, Ph.D, Associate Professor 
The State Key Laboratory of Automotive Safety and Energy

Department of Automotive Engineering, Tsinghua University

Beijing 100084, P.R.China

Tel: +86-10-62785599, Fax: +86-10-62784616, Email: 1ct@tsinghua.edu.cn

Xiaotian Xu, Postgraduate

The State Key Laboratory of Automotive Safety and Energy

Department of Automotive Engineering, Tsinghua University

Beijing 100084, P.R.China

Tel: +86-1521-056-0430 